\title{
Acciones de oro y patriotismo económico: enfoque nacional, desarrollo europeo*
}

\author{
Golden Shares and Economic Patriotism: \\ National Approach, European Development \\ Jérémie Houet \\ Doctor en Derecho por la Universidad de Paris X, miembro \\ del Centre d'études juridiques européennes et comparées (CEJEC)
}

doi: 10.18543/ced-54-2016pp203-231

Sumario: I. Introducción. 1.1. Contexto. 1.2. Libertades de circulación y las acciones de oro. 1.3. Plantilla de análisis de las acciones de oro. 1.4. Margen de maniobra y patriotismo económico.-II. El mantenimiento delicado de las acciones de oro nacionales. 2.1. La persistencia de acciones de derechos especiales. 2.1.1. Persistencia de derechos especiales en algunos sectores. 2.1.2. Economías en transición. 2.1.3. Procedimiento previo. 2.1.4. Nuevos regímenes inciertos. 2.2. La continuación del control por parte de la Comisión. 2.2.1. Continuidad de los controles estrictos de la Comisión. 2.2.2. Regímenes validados por la Comisión.-III. La necesidad de una directiva sobre las acciones de oro. 3.1. La oportunidad de una directiva de las acciones de oro. 3.1.1. Acciones de oro organizadas por el derecho de la UE. 3.1.2. Abastecimiento energético. 3.1.3. Transporte aéreo de pasajeros. 3.1.4. Defensa. 3.1.5. Telecomunicaciones. 3.2. La aplicación de una directiva sobre las acciones de oro. 3.2.1. Competencia. 3.2.2. El proceso Lamfalussy. 3.2.3. Armonización positiva. 3.2.4. Aplicación en el marco de una directiva de las acciones de oro.-IV. Conclusión.

Resumen: El mecanismo de las acciones de oro permite a los Estados miembros a retener algún control sobre las empresas nacionales que operan en sectores estratégicos. A pesar de que está estrictamente controlada tanto por la Comisión y la Corte, la crisis actual pone este dispositivo al primer plano. Los Estados miembros son propensos a reapropiarse por medio de desviados recursos. Sus intentos revelan un malestar más profundo; el miedo a las inversiones extranjeras en esos sectores. Esto plantea la cuestión de la oportunidad de un mecanismo común para supervisar estas inversiones extranjeras. Por lo tanto, la respuesta a la crisis debe superar el nivel nacional para situarse a escala europea. ¿Podría el patriotismo económico entonces ser supranacional?

Palabras clave: golden shares, acciones de oro, derechos especiales, libertades de circulación, inversiones extranjeras, sectores estratégicos.

* Recibido el 21 de septiembre de 2015, aceptado el 27 de enero de 2016. 
Abstract: The "golden shares» mechanism allows Member States to retain some control over domestic companies operating in strategic sectors. Although it is strictly controlled by both the Commission and the Court, the current crisis brings this instrument back to center stage. Member States are likely to re-use it through diverted resources. Their attempts reveal a deeper malaise; the fear of foreign investments in these sectors. This raises the question of the opportunity of a common mechanism for monitoring these foreign investments. The answer to the crisis is therefore proposed at the level of the European Union, not just at the Member States'. Could economic patriotism then be supranational?

Keywords: golden shares, special rights, freedoms of movement, foreign investment, strategic sectors.

\section{Introducción}

\subsection{Contexto}

Las políticas económicas aplicadas tanto en los EEMM (Estados Miembros) como a nivel de la UE (Unión Europea) deben crear un clima favorable para el crecimiento y el desarrollo de los negocios transfronterizos. Estos últimos deben estar en condición de hacer frente a la competencia en igualdad de condiciones. Capaces de basarse en regímenes jurídicos adecuados que les permitan practicar sus actividades en toda la UE, e incluso fuera de ésta. Deben, además, poder invertir en el extranjero así como atraer capitales para financiar su desarrollo.

Ahora bien, en algunos casos, las empresas en cuestión juegan un papel estratégico en la defensa, el transporte o en la salud pública de una economía nacional. En otros, se trata simplemente de empresas importantes, de «campeones nacionales» cuyos gobiernos desean que sigan manteniendo su identidad nacional. El mecanismo de golden shares (acciones de oro) permitió atender las demandas de los EEMM que deseaban mantener el control sobre las empresas, en especial de aquellas que iban privatizando paulatinamente $^{1}$. A corto y mediano plazo, el mecanismo de esas acciones específicas les permite controlar la dirección estratégica de la empresa.

${ }^{1}$ Una acción de oro es un título representativo de una parte del capital de una empresa, a la cual se adhieren diferentes derechos especiales. Estos permiten tener al titular, facultades con una amplitud tal que solo las participaciones mayoritarias normalmente le hubieran podido asegurar, tanto en la estructura accionarial de la empresa, como en su gestión. 


\subsection{Libertades de circulación y las acciones de oro}

Si no se cumplen determinados requisitos, dicho mecanismo se verá invalidado por la Comisión y por el TJUE (Tribunal de Justicia de la Unión Europea). La jurisprudencia de acciones de oro está, en este sentido, llena de lecciones ${ }^{2}$. En primer lugar, se ha observado al realizar el control de dicho mecanismo que el TJUE ha sabido aventurarse progresivamente en el terreno de un razonamiento basado en el «capital», debido a los particularismos macro-económicos de los movimientos de capitales ${ }^{3}$. En el marco de las acciones de oro, y con el fin de cualificar correctamente las restricciones nacionales impugnadas, ha sido esencial buscar si el contenido de la medida en cuestión limita la libre circulación de capitales o la libertad de establecimiento, de forma totalmente independiente una de la otra.

${ }^{2}$ La lista de asuntos sobre las acciones de oro es la siguiente: Asunto C-58/99, Comisión/República Italiana, Sentencia del TJUE de 23 de mayo de 2000, Rec. 2000, p. I-3811; Asunto C-503/99, Comisión/Reino de Bélgica, Sentencia del TJUE de 4 de junio de 2002, Rec. 2002, p. I-4809; Asunto C-367/98, Comisión/República Portuguesa, Sentencia del TJUE de 4 de junio de 2002, Rec. 2002, p. I-4731; Asunto C-483/99, Comisión/República Francesa, Sentencia del TJUE de 4 de junio de 2002, Rec. 2002, p. I-4781; Asunto C-98/01, Comisión/Reino Unido de Gran Bretaña y de Irlanda del Norte, Sentencia del TJUE de 13 de mayo de 2003, Rec. 2003, p. I-4641; Asunto C-463/00, Comisión/Reino de España, Sentencia del TJUE de 13 de mayo de 2003, Rec. 2003, p. I-4581; Asunto C-174/04, Comisión/República Italiana, Sentencia del TJUE (Sala Primera) de 2 de junio de 2006, Rec. 2006, p. I-4933; Asuntos C-282/04 y C-283/04, Comisión/Reino de los Países Bajos, Sentencia del TJUE (Sala Primera) de 28 de septiembre de 2006, Rec. 2006, p. I-9141; Asunto C-112/05, Comisión/Alemania, Sentencia del TJUE (Gran Sala) de 23 de octubre de 2007, Rec. 2007, p. I-8995; Asuntos C-463/04 y C-464/04, Federconsumatori y otros, Sentencia del TJUE (Sala Primera) de 6 de diciembre de 2007, Rec. 2007, p. I-10419; Asunto C-326/07, Comisión/República Italiana, Sentencia del TJUE (Sala Tercera) de 26 de marzo de 2009, Rec. 2009, p. I-2291; Asunto C-171/08, Comisión/República Portuguesa, Sentencia del TJUE (Sala Primera) de 8 de julio de 2010, Rec. 2010, p. I-6817; Asunto C-543/08, Comisión/República Portuguesa, Sentencia del TJUE (Sala Primera) de 11 de noviembre de 2010, Rec. 2010, p. I-11241; Asunto C-212/09, Comisión/República Portuguesa, Sentencia del TJUE (Sala Primera) de 10 de noviembre de 2011, Rec. 2011, p. I-10889; Asunto C-244/11, Comisión/República Helénica, Sentencia del TJUE (Sala Cuarta) de 8 de noviembre de 2012, ECLI:EU:C:2012:694; Asunto C-95/12, Comisión/Alemania, Sentencia del TJUE (Gran Sala) de 22 de octubre de 2013, ECLI:EU:C:2013:676; Asuntos C-105/12 a C-107/12, Essent, Sentencia del TJUE (Gran Sala) de 22 de octubre de 2013, ECLI:EU:C:2013:677.

${ }^{3}$ LUBY, M., «Chroniques» en Journal de Droit International, 1997, n. 2, pp. 556 y ss., con respecto al asunto C-484, Svensson y Gustavsson, Sentencia del TJUE de 14 noviembre 1995, Rec. 1995, p. I-3955; M.A., P. de, «La vinculación de la concesión de ayudas públicas a que la entidad que las financia esté autorizada en el Estado miembro es un obstáculo al libre movimiento de capitales» en La ley - Unión Europea, n. ${ }^{\circ}$ 3974, 1996, pp. 14-15. 
Por tanto, la cuestión de la acción específica, con respecto a la intención del inversor, engloba básicamente dos situaciones. Para aquel que pretende una participación destinada a controlar y ejercer de manera efectiva sus derechos, las facultades especiales de las autoridades públicas presumirán una intromisión notable en la autonomía organizativa de la empresa. Tales facultades públicas no crean un obstáculo similar para el accionista minoritario, sobre todo interesado en la rentabilidad financiera de su participación y sin influencia práctica en los órganos de gestión de las empresas. En la primera situación, estas relaciones se refieren al derecho del establecimiento y, de manera subsidiaria, a la libre circulación de capitales. En la segunda situación, por el contrario, es más apropiado dar preferencia a la segunda libertad que a la primera ${ }^{4}$.

A pesar de esta separación aparente de las dos libertades, las restricciones de la libre circulación de capitales y de la libertad de establecimiento derivadas de la acción específica, y de los derechos especiales que están vinculados, no significan necesariamente que el mecanismo viole el derecho de la UE. Un cierto número de excepciones permiten, bajo ciertas condiciones, legitimar la injerencia de los principios que contiene. El análisis económico propuesto por la Comisión no siempre es convincente (especialmente porque no tiene en cuenta los aspectos sociales positivos de las acciones de oro), de manera que tenemos el derecho de interrogarnos si la institución busca simplemente, y por todos los medios, lograr sus objetivos, es decir, la condena de los derechos especiales instaurados por los EEMM.

\subsection{Plantilla de análisis de las acciones de oro}

Los EEMM disponen de múltiples motivos para justificar los derechos especiales. Estos últimos, sin embargo, deberán apreciarse con referencia a su pertinencia y a su proporcionalidad con respecto al objetivo perseguido ${ }^{5}$. Cuanto más se aleje el motivo de las verdaderas intenciones de los EEMM, más le será difícil pasar el control del TJUE. Conviene señalar que el motivo mencionado por los EEMM, no debe ser redundante; debe existir un verdadero vínculo entre el método elegido y el objetivo perseguido.

${ }^{4}$ DE LA SERNA BILBAO, M.N., «Comentario a la jurisprudencia del Tribunal de Justicia de la Comunidad Europea en relación con las denominadas «acciones de oro»; las restricciones a las libertades de la libre circulación de capitales y de establecimiento» en Revista Española de Derecho Europeo, n. ${ }^{\circ}$ 7, 2003, pp. 529 y ss.

5 GIPPINI-FOURNIER, E., y RODRÍGUEZ MÍGUEZ, J.-A., «Golden shares en la Comunidad Europea: ¿fin de la edad dorada?» en Gaceta Jurídica de la C.E. y de la Competencia, $.^{\circ} 220,2002$, pp. 38-62. 
Asimismo, ningún otro mecanismo menos restrictivo puede remplazar los derechos especiales establecidos por los EEMM. Es preciso constatar aquí que el mecanismo de autorización previa, muy frecuentemente utilizado, puede ser sustituido por el de la declaración a priori, y mejor aún, $a$ posteriori.

Por último, el mecanismo debe estar basado en criterios objetivos, es decir, enfocando una meta explícita en términos precisos y claros, con reglas de juego transparentes conocidas con anterioridad por los interesados. Estas deben ser cuestionadas por las decisiones específicas y no fundamentales en la vida de la empresa. Cualquier persona afectada por tal medida restrictiva, debe disponer de vías judiciales para restablecer sus derechos.

Esta plantilla de análisis constituye un mínimo al que deben ajustarse los EEMM. Por supuesto, estos últimos son libres de añadir aún más flexibilidad en los regímenes con el fin de garantizar mejor protección de las libertades de circulación. Si estos criterios no se cumplen correctamente, o si los EEMM tratan de remplazarlos por otros, como el carácter temporal de los derechos especiales, estos no estarán justificados. Por ello, el margen de maniobra del que disponen los EEMM es, en realidad, particularmente reducido $^{6}$.

\subsection{Margen de maniobra y patriotismo económico}

En definitiva, el análisis jurídico de las acciones de oro tiende a confirmar lo que las instituciones de la UE concedieron con una mano, por medio de la aceptación de motivos cada vez más numerosos justificando las excepciones a las libertades de circulación, y retiraron con la otra mano por medio de la aplicación rigurosa del control de estas justificaciones ejerciéndose por medio de una lectura estricta de su pertinencia y proporcionalidad. El examen del marco y del ejercicio del control de las acciones específicas a través de la jurisprudencia del TJUE ha permitido revelar que el mecanismo no está prohibido per $\mathrm{se}^{7}$. Por consiguiente, su práctica no está com-

${ }^{6}$ GARAU SOBRINO, F., «La incompatibilidad con el derecho comunitario de la normativa Española sobre inversión en empresas públicas privatizadas. Ultimo acto y telón» en Gazeta Jurídica, n. ${ }^{\circ} 225,2003$, pp. 56 y ss.; URREA CORRES, M., «El régimen de autorizaciones administrativas en la empresas privatizadas» en Revista de Derecho Comunitario Europeo, $.^{\circ} 15,2003$, pp. 683 y ss.

7 JIMENEZ-BLANCO, A., «El Tribunal de justicia contra la Ley Rato» en Noticias de la UE, n. ${ }^{\circ}$ 297, 2009, pp. 127 y ss.; REDONDO TRIGO, F., «La toma de participación en empresas españolas energéticas a la luz de la reciente jurisprudencia del Tribunal de Justicia de las Comunidades Europeas» en Revista Critica de Derecho Inmobiliario, n. ${ }^{\circ}$ 712, 2009, pp. 959 y ss. 
pletamente eliminada principalmente con respecto al contexto actual de la crisis financiera.

Dicho contexto incitó a los EEMM a replegarse hacia sus economías domésticas. Los riesgos geopolítico y económico provocados por las participaciones de capital de los nuevos inversores, en particular de los fondos soberanos, y también de los operadores clásicos, en las empresas nacionales estratégicas, han suscitado movimientos de patriotismo económico transnacional. Dichos movimientos han aparecido en los diferentes países de la UE, incluso en algunas regiones nacionales. El objetivo de estos movimientos consiste, principalmente, en mantener el empleo y las empresas en territorio nacional, amenazados por las participaciones en el capital de las inversiones extranjeras que las autoridades públicas, según la opinión pública, deben controlar. El discurso político debe, sin embargo, materializarse por medio de un arsenal jurídico eficaz.

Ahora bien, en la UE, los EEMM ya no conceden la posibilidad de utilizar los recursos de acciones tradicionales para controlar ese tipo de participaciones. Utilizarán, en su momento, métodos discrecionales para controlar los flujos de los capitales extranjeros. Las herramientas jurídicas se han desarrollado pero algunas veces se ha puesto en tela de juicio su legalidad. Para proteger a sus empresas, las autoridades públicas nacionale han mantenido y renovado sus prácticas de las acciones específicas (II). Ello ha sido así porque, si bien la jurisprudencia de las acciones de oro condena algunos de sus usos, no es menos cierto que deja un margen de maniobra a las autoridades públicas para recurrir al mecanismo de los derechos especiales. De la misma manera, la variedad de estos mecanismos, entre los EEMM, nos lleva a preguntarnos si la armonización de la práctica y la clarificación de su uso no deberían, a partir de ahora y en el futuro, canalizarse a través de reglas claras establecidas por la Comisión (III).

\section{El mantenimiento delicado de las acciones de oro nacionales}

La voluntad de las autoridades públicas de continuar utilizando las acciones específicas se manifiesta de dos formas. Por una parte, mediante el mantenimiento tradicional de derechos especiales en algunas empresas y, por otra, a través de la adaptación y la adecuación de normas que organicen el control de las inversiones extranjeras (1). Por tanto, el control de la Comisión sigue su análisis, debiendo renovarse frente a la ingenuidad con la que los EEMM utilizan, o incluso abusan, de sus margenes de maniobra (2). 


\subsection{La persistencia de acciones de derechos especiales}

\subsubsection{Persistencia de derechos especiales en algunos sectores}

En su Comunicación de 2005, la Comisión identificó una multitud de derechos especiales en las empresas que operan en los sectores importantes de la economía. Se trata de empresas encargadas de la prestación de servicios públicos en las telecomunicaciones, la electricidad, el gas, la energía, y también en los servicios postales, la banca e incluso en los seguros ${ }^{8}$. En la última década, un gran número de derechos especiales han sido, o están en curso de ser abolidos o reducidos.

No obstante, hemos de considerar que las autoridades públicas tratan de mantener algunos mecanismos en sectores que consideran estratégicos. Ahora bien, la calificación como derecho de tal o cual sector como «estratégico» resulta problemática. En efecto, existe una multitud de sectores que se ubican en la frontera de esta terminología. Hay además muchas empresas cuyas actividades pueden, a veces, encajar en la definición de ámbito estratégico, y a veces no, aumentando o disminuyendo respectivamente el ámbito de tales sectores. Dado que la demarcación a nivel nacional resulta problemática por su falta de claridad, la obtención de una posición común a nivel europeo, se convierte en una tarea laboriosa. La demarcación de un «perímetro estratégico de soberanía» ${ }^{9}$ es una actividad ardua. No obstante, parece que hay unanimidad para integrar en ese perímetro a los sectores de la defensa y de la energía. Cabe señalar a este respecto que la mayoría de las acciones de oro aún existentes se encuentran en las empresas de estos sectores, que podrían ser percibidos como un conjunto común mínimo.

Así lo demostró la negociación entre la Comisión y Luxemburgo con respecto a la empresa de satélite SES. En 2005, la Comisión ha decidido concluir el procedimiento de infracción relativo al contrato de concesión celebrado entre el gobierno luxemburgués y la empresa de satélites SES Astra. Dicho contrato permitió al Estado luxemburgués bloquear determinadas tomas a de participaciones en la empresa SES Astra, y en su cartera matriz SES Global. Las disposiciones pertinentes han sido modificadas a través de un nuevo contrato de concesión adoptado por el gobierno luxemburgués y la empresa SES Astra. Este nuevo contrato limita el de-

${ }^{8}$ Comunicación de la Comisión Europea, de 22 de julio 2005, Special rights in privatised companies in the enlarged Union-a decade full of developments, disponible en Internet, European Commission staff working document: http://ec.europa.eu/internal_market/capital/ docs/privcompanies_en.pdf (última consulta 18/01/2016).

9 DEBELCQUE, E., Quel patriotisme économique?, Presses Universitaires de France, Paris, 2008. 
recho del gobierno a oponerse a la adquisición de participaciones en SES Astra por un accionista que, por razones de orden público o de seguridad pública, representaría una amenaza seria para el funcionamiento de una red de satelite ${ }^{10}$.

\subsubsection{Economías en transición}

Si los derechos especiales desaparecen progresivamente de los EEMM antiguos, todavía están presentes en determinadas economías en transición. Por razones ligadas a las fechas y a la magnitud del proceso de privatización en los EEMM más recientes, tales derechos tienden a desaparecer. Por ejemplo, aunque el informe de la Comisión de 2005 no mencionaba ninguna acción de oro en las manos del gobierno estonio, éste último tenía una en la empresa de ferrocarriles AS Esti Raudtee, en el momento de su adhesión el 1 de Mayo 2004. El Estado disponía de un derecho de veto en las asambleas de los accionistas y en las del consejo de administración, así como la posibilidad de nombrar representantes del Estado en el consejo. Esos derechos especiales infringen la libre circulación de los capitales y la libertad de establecimiento de la $\mathrm{UE}^{11}$. En vez de verse condenado por la Comisión y el TJUE, y en la medida en que consideraba esta sociedad como estratégica, el Estado estonio decidió adquirir la mayor parte del capital y, posteriormente, su totalidad. De esta manera recobró el control de la empresa, evitando la sanción que se le habría impuesto de haber seguido siendo un accionista minoritario.

\subsubsection{Procedimiento previo}

A fin de permitir a los EEMM adaptarse de manera voluntaria a los imperativos del derecho de la UE, la Comisión entabla generalmente un proce-

10 Ver el comunicado de prensa de la Comisión Europea del 20 de diciembre 2005, IP/05/1636, en donde se especifica que «las restricciones relativas a las acciones de SES Astra, estipulan que al menos $79,9 \%$ de estas, deberán estar controladas en todo momento, por SES Global, mientras dure la concesión. Las restricciones relativas a las acciones de SES Global estipulan, por su parte, que el Gobierno luxemburgués tiene un derecho de oposición a la tenencia, directa o indirecta, por un accionista o varios accionistas que actúen de concierto, de más de $20,1 \%$ de dichas acciones. El gobierno puede ejercer dicho derecho solo por determinadas razones de interés público», disponible en Internet, European Commission Press Database Release: http://europa.eu/rapid/press-release_IP-05-1636_en.htm?locale=en (última consulta 18/01/2016).

11 KUZNETSOV, I., «Special rights in EU privatised companies: a few remarks on the Estonian experience» en GOLDAMMER, Y. (coor.), The long road of smaller countries into the enlarged European Union, Eugrimas, Vilnius, 2006, pp. 96 y ss. 
dimiento previo que le permite interactuar con las autoridades públicas para clarificar los obstáculos de la ejecución de derecho de la UE ${ }^{12}$.

Así sucedió, por ejemplo, en el caso de Bulgaria ${ }^{13}$, miembro de la UE desde 2007. El Gobierno búlgaro no podía ignorar las primeras sentencias del TJUE sobre las acciones de oro. En tanto que país candidato a la adhesión, Bulgaria debía integrar el acervo comunitario y armonizar sus leyes y normas en consonancia con dicho acervo. Si bien tal ejercicio parecía haberse llevado a cabo, se trataba, la mayor parte del tiempo, de una simple declaración del gobierno justificando el mantenimiento de una acción específica por razones de «interés público ». Este fue el caso en las privatizaciones de la empresa petroquímica Neftochim Burgas (cedida finalmente a la empresa rusa Lukoil), de las empresas Bulgartabak Holding y Bulgarian Telecommunications Company, que ostentaban, respectivamente, el monopolio del tabaco y el de las telecomunicaciones.

Aunque desde su adhesión en 2007 Bulgaria tuvo la posibilidad de cumplir las normas del TFUE, aboliendo los derechos especiales ilegales, no puso demasiado empeño en tal tarea. En una decisión de marzo $2013^{14}$, la Comisión ordenó al gobierno búlgaro suprimir los mecanismos susceptibles de restringir la libre circulación de capitales de la ley búlgara, con respecto a la privatización y el control después de la privatización, que atribuía derechos especiales a las autoridades públicas ${ }^{15}$. Dicha voluntad de dialogo de la Comisión con las autoridades públicas permitió anticipar las condenas posibles y acompañar las economías en transición a fin de respetar el derecho de la UE.

\subsubsection{Nuevos regímenes inciertos}

Las participaciones de los inversores extranjeros están ahora sujetas a un régimen de declaración o de autorización ex ante, o incluso de oposición ex post. Eso régimen se refiere, en general, a sectores de actividad especí-

12 Ver Comisión Europea, Commission staff working document on the free movement of capital in the EU, del 15 abril 2013, SWD (2013) 146 final, disponible en Internet, European Commission staff working document: http://ec.europa.eu/finance/capital/docs/reports/2013market-monitoring-working-document_en.pdf (última consulta 18/01/2016).

${ }^{13}$ MILLER, L., y BOCK, C., «Golden Shares and EU Accession: Bulgaria's Balancing Act» en Journal of European Affairs, n. $^{\circ}$ 1, 2003, vol. 1.

14 Ver Comisión Europea, MEMO/13/261, de 21 marzo 2013, disponible en Internet, European Commission Press Database Release: http://europa.eu/rapid/press-release_ MEMO-13-261_en.htm (última consulta 18/01/2016).

15 Dicha ley establecía que las autoridades públicas búlgaras podían obtener hipotecas y otras garantías, no solamente en los bienes del accionista indicado por el gobierno, sino también en el conjunto de la empresa privatizada. 
ficos o sectores donde el concepto de interés general prevalece. Estos están vinculados con los intereses vitales y esenciales de la defensa nacional, del orden público y de la seguridad pública, incluso de la salud pública. No faltan ejemplos de tales reglamentaciones entre los EEMM de la UE. Si algunas plantean incertidumbres, otras, en principio, parecen haber obtenido la aprobación de la Comisión ${ }^{16}$.

Italia.-Tras su condena ${ }^{17}$, el régimen italiano ha sido modificado en marzo de 2012 por el decreto-ley 21/2012 ${ }^{18}$, tal como quedó enmendado y ratificado por la ley del 11 de mayo $2012^{19}$, que se aplica a cualquier empresa que posee o controla activos estratégicos en los asuntos de defensa, seguridad nacional, energía, transportes y telecomunicaciones, independientemente del hecho de que el Estado sea accionista o no. Por ello se prevé que cualquier acto, resolución o transacción adoptado por los órganos sociales de las empresas afectadas susceptible de afectar al funcionamiento interno, a la propiedad o a la disponibilidad de los activos estratégicos, deberá ser notificado al gobierno italiano ${ }^{20}$. Se prevé un periodo de stand-still de 15 días. En la medida en que se cumplan las condiciones de ejercicio, el gobierno dispone de dos posibilidades: someter la transacción a condiciones específicas, u oponer su veto a la transacción, como medida de último recurso. No obstante se espera, que este derecho de veto se ejercerá solamente en contra de las empresas que operan en los sectores mencionados, y solo se dirigirá a los inversores que no son miembros de la UE. Asimismo se ha previsto una condición de reciprocidad. En efecto, sin perjuicio de la facultad de oposición de la cual dispone el gobierno italiano en una transacción, los inversores no miembros de la UE, que deseen adquirir una participación en una de las empresas estratégicas, solo estarán autorizados en el caso de que su país de origen permita de igual manera a los ciudadanos ita-

${ }^{16}$ No es nuestra intención discutir aquí el conjunto de reglamentaciones existentes en la UE, sino destacar las más simbólicas de sus ámbitos de aplicación. En el Reino Unido, por ejemplo, no existe ninguna legislación sobre los inversores directos extranjeros. No obstante una disposición de la Ley aplicable a las concentraciones de 2002 (Entreprise Act) permite al gobierno rechazar las concentraciones de empresas no británicas si los intereses del país están amenazados.

17 COCCIOLO, E., y PADRÓS REIG, C., «La doctrina del TJCE sobre las acciones de oro: Entre la construcción del Mercado interior y los retos de la intervención pública en los mercados globales. Reflexiones acerca de la STJCE de 26 de marzo de 2009 (TJCE 2009, 70), asunto C-326/07» en Revista Española de Derecho Europeo, n..$^{\circ} 33$, 2010, pp. 123-156.

${ }_{18}$ Decreto-ley n. ${ }^{\circ} 21$ del 15 marzo 2012.

19 Ley n. 56 del 11 mayo 2012.

${ }^{20}$ BONFITTO, V., «Italian M\&A: new "golden share” rules explained», Julio 2012, disponible en Internet, International Financial Law Review: http://www.iflr.com/Article/3057594/Italian-M-A-newGolden-Share-rules-explained.html (última consulta 18/01/2016). 
lianos convertirse en accionistas de las empresas de su país en condiciones similares. No obstante, dicha condición de recíprocidad se supeditará a los tratados internacionales celebrados por Italia o por la UE. Por último, debe señalarse que el nuevo régimen no deroga las normas existentes, por ejemplo con respecto a la posibilidad, para las empresas cuyo Estado es accionista y que opera en los sectores estratégicos de introducir en sus estatutos un límite a la adquisición de una participación por cualquier individuo o empresa hasta del 5\% del capital. Sólo cabe ser escépticos frente a este nuevo régimen que, si se aplica de forma no proporcional, se verá seguramente sancionado por la Comisión y por el TJUE. Conviene señalar que el derecho de veto acompañado de la condición de reciprocidad, aunque se aplique solamente a los inversores de Estados terceros estará sujeto al principio de la libre circulación de capitales dado que esta última libertad, hay que recodarlo, beneficia de hecho a los ciudadanos y empresas de estados terceros, contrariamente a las otras libertades de circulación.

Francia.-Tras una sentencia del TJUE de 2002, el régimen francés de autorización previa tuvo que ser modificado. El gobierno debía elegir entre abandonar pura y simplemente el mecanismo o modificar las modalidades de aplicación del régimen. Optó por el segundo enfoque, aprobando el decreto . $^{\circ}$ 2003-196 del 7 de marzo 2003, derogado por el decreto $n .^{\circ} 2005$ 1739 de 30 de diciembre de $2005^{21}$, conocido prácticamente como «decreto anti-OPA» 0 «decreto del patriotismo económico» ${ }^{22}$. Dicho decreto estableció una lista de sectores estratégicos vinculados al orden y la seguridad públicos así como a la defensa nacional, para los cuales los inversores provenientes de un Estado miembro de la UE o del extranjero ${ }^{23}$ quedan sujetos a una autorización previa.

De acuerdo con el decreto, una inversión proveniente de un tercer Estado constituye « el hecho de adquirir el control en el sentido del artículo L.

${ }^{21}$ Decreto n. ${ }^{\circ}$ 2005-1739, de 30 diciembre 2005, que regula las relaciones financieras con el extranjero y relativo a la aplicación del artículo L. 151-3 del código monetario y financiero, JORF n. 304 del 31 diciembre 2005, p. 20779.

22 Ver, por ejemplo, MARSON, G., «Le décret de "patriotisme économique" réglementant les relations financières avec l'étranger» en Contrats Concurrence Consommation, Marzo 2006, n. ${ }^{\circ} 3$.

${ }^{23} \mathrm{El}$ inversor «comunitario» puede ser una persona física, ciudadano de un Estado miembro de la UE o del EEE, o una empresa que tenga su domicilio social en uno de esos Estados o, finalmente, una persona física de nacionalidad francesa residente en ese país. Nos referimos también a las inversiones directas o indirectas, lo que extiende considerablemente su ámbito de aplicación en la medida en que una empresa establecida en Francia o en un Estado miembro de la UE o del EEE, controlada por personas ubicadas fuera de la UE o del EEE, se considera inversor no «comunitario» en el sentido del artículo R. 153-1 del Código Monetario y Financiero. 
233-3 del Código de Comercio $»^{24}$, incluyendo el control conjunto, «de adquirir directa o indirectamente todo o una parte de la rama de actividad de una empresa cuyo domicilio social está establecido en Francia» ${ }^{25}$, o « de rebasar el límite de 33,33\% de la posesión directa o indirecta del capital o de los derechos de voto de una empresa cuyo domicilio social esté ubicado en Francia ${ }^{26}$, esto es, la adquisición de una minoría de bloqueo. La determinación de las inversiones provenientes de un Estado miembro de la UE es más estrecha, en la medida en que sólo afecta a la adquisición del control, en el sentido del artículo L. 233-3 del Código de Comercio, y a la adquisición de una rama de actividad. De manera general, las inversiones que entren en el ámbito del decreto, no pueden, por así decirlo, esquivar la demanda de autorizacion ${ }^{27}$. Sin embargo es posible formular una petición previa escrita para la solicitud de autorización «para determinar si una inversión concreta está sujeta a un procedimiento de autorización» ${ }^{28}$.

La publicación del decreto no fue sin embargo bien recibida por la Comisión. Esta envió sucesivamente a Francia una carta de petición de información el 20 de enero 2006, una carta de emplazamiento el 4 de abril y finalmente un dictamen motivado el 12 de octubre de 2006, última etapa antes de una eventual acción ante el TJUE. El gobierno francés se apresuró entonces a proporcionar explicaciones y justificaciones a la Comisión, argumentando principalmente que era «normal impedir a las empresas no europeas con una filial en la UE "ir de compras" en Francia sin control alguno» ${ }^{29}$. Desde entonces, la Comisión actúa con benevolencia y no ha dado muestras de querer continuar el procedimiento ante el TJUE.

${ }^{24}$ Decreto n. $^{\circ}$ 2005-1739, op. cit., artículo 2.

25 Ibid.

${ }^{26}$ Ibid.

${ }^{27}$ La autorización es válida únicamente en tres situaciones, incluso si la inversión prevista se efectúa entre dos empresas que pertenecen al mismo grupo, salvo si tiene por objeto trasferir al extranjero todo o una parte de la rama de actividad o mientras el ministro encargado de la economía no haya respondido a la solicitud de autorización en los dos meses siguientes a su recepción.

${ }^{28}$ Decreto n. ${ }^{\circ} 2005-1739$, op. cit., artículo 4.

29 GAUDIN, C., «La bataille des centres de décision : promouvoir la souveraineté économique de la France à l'heure de la mondialisation», informe n. ${ }^{\circ} 347$ hecho en nombre de la Mission commune d'information centre de décision économique, 22 junio 2007, disponible en Internet, Sénat: http://www.senat.fr/rap/r06-347-1/r06-347-1.html (última consulta 18/01/2016). El autor explica que «en cuanto a las empresas cuya parte marginal del volumen de negocios es realizado en las actividades sensibles, el Gobierno francés hace valer que el decreto prevé, para las empresas de la UE, un examen de "la rama estratégica" mencionada y "no del conjunto de la empresa". Por otra parte, el gobierno puede examinar una toma de control "sin bloquear la inversión", pidiendo al inversor "compromisos limitados al único establecimiento afectado". Finalmente, dicho gobierno reconoce que el sector de los casinos podría ser excluido del ámbito del decreto.» 
Después de la tentativa de toma de participación en el capital de Alstom por parte de General Electric al inicio de 2014, el decreto ha sido actualizado el 14 de mayo de 2014 para ampliar los nuevos sectores del procedimiento de autorización, y tomar en cuenta las actividades esenciales para la garantía de los intereses del país, en materia de orden público, seguridad pública o defensa nacional, para garantizar principalmente la continuidad del suministro de electricidad, de gas, de hidrocarburos y de otras fuentes de energía, así como la explotación de las redes y de los servicios de transporte y de telecomunicaciones ${ }^{30}$.

Las discusiones entre la Comisión y Francia con respecto al decreto pa-

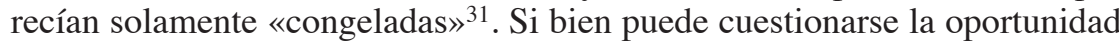
del decreto respecto de determinados sectores, es poco probable que la Comisión impugne los sectores añadidos por Francia en 2014, en la medida en que éstos se inspiran exactamente de la jurisprudencia del TJUE.

Alemania.-Tras la decisión del TJUE en 2007, se han hecho modificaciones a la ley $\mathrm{VW}^{32}$. Cabe recordar los tres derechos especiales debatidos en la sentencia: la posibilidad del Estado federal y de la Baja Sajonia, siempre que sean accionistas de Volkswagen AG, de designar cada uno, dos representantes en calidad de miembros del consejo de vigilancia (artículo 4, párrafo 1 de la ley); el límite de los derechos de voto de cada accionista de $20 \%$ del capital social (artículo 2, párrafo 1); y la fijación de la minoría de bloqueo de $20 \%$ para las decisiones más importantes de la asamblea general (artículo 4, párrafo 3). El TJUE declaraba, en el fallo de su sentencia, que Alemania contravenía a la libre circulación de los capitales manteniendo en vigor el artículo 2, párrafo 1, « en relación con »el artículo 4, párrafo 3, de la ley del 21 de julio $1960^{33}$. La Comisión esperaba de este modo ver desaparecer cada uno de los derechos.

No obstante, el 10 diciembre 2008, la ley VW fue revisada, y se conservó una cláusula, el artículo 4, párrafo 3. Dicho artículo, ligeramente modificado, prevé que a partir de ahora y en lo sucesivo, la Baja Sajonia dispondría de un estatuto de minoría de bloqueo, que normalmente se reserva a los accionistas que tienen más de $25 \%$ de los derechos de voto. Para la Comisión, el hecho de que la parte afectada del mecanismo de la sentencia de 2007 contenuviera los términos «en relación con», no excluía el ca-

30 Decreto n. ${ }^{\circ}$ 2014-479, del 14 mayo 2014, relativo a las inversiones extranjeras sujetas a la autorización previa.

31 Ibid.

32 RODRÍGUEZ MIGUEZ, J.A., y PADRÓS REIG, C., «Las acciones de oro, el derecho de sociedades y el mercado interior. Reflexiones a propósito de la STJCE sobre la Ley Volkswagen» en Gaceta Jurídica de la C.E. y de la Competencia, n. ${ }^{\circ}$ 8, 2009, pp. 47-75.

33 Asunto C-112/05, op . cit., parte dispositiva de la sentencia, alinéa 1. 
rácter ilegal de cada una de las disposiciones controvertidas. En cambio, el gobierno alemán sostiene que la parte dispositiva de la sentencia no debía entenderse referido a las tres disposiciones controvertidas consideradas por separado $^{34}$. En ausencia de una respuesta satisfactoria, la Comisión recurrió entonces al TJUE con respecto a la base del artículo 260 TFUE $^{35}$.

Para el Abogado general Wahl, en sus conclusiones presentadas el 29 de mayo 2013, la cuestión es exclusivamente determinar el sentido de la parte dispositiva de la decisión a la luz de sus motivos. Por lo que se considera que el argumento del gobierno alemán es correcto ${ }^{36}$. En su sentencia del 22 octubre de 2013, el TJUE confirmó este enfoque, considerando que «la motivación de la sentencia Comisión/República Federal de Alemania, antes citada, confirma por lo tanto la conclusión basada en el punto 1 del fallo de dicha sentencia de que el incumplimiento declarado por el TJUE no derivaba del artículo 4, apartado 3, de la Ley Volkswagen, considerado aisladamente, sino únicamente de la combinación de esta disposición con el artículo 2, apartado 1, de la mencionada Ley $»^{37}$. En definitiva, la cuestión es saber si la minoría de bloqueo que confiere el artículo 4, párrafo 3 de la ley VW, modificada constituye una violación del derecho de la UE, y debe decidirse en el marco de un nuevo procedimiento fundado en el artículo 258 TFUE. El mantenimiento de dicho derecho especial por parte del länder de Baja Sajonia es, por consiguiente, susceptible de una condena posterior de la Comisión, la protección de un interés nacional a través de la empresa Volkswagen ya que es muy ambigua.

Asimismo, en respuesta a las preocupaciones vinculadas al aumento de la toma de participaciones de los fondos soberanos en las empresas alema-

34 Alemania, en una carta del 17 diciembre 2008, transmitió a la Comisión una propuesta que consistía en solicitar al TJUE una petición interpretativa conjunta de la sentencia inicial Comisión/República Federal de Alemania, conforme a los artículos 43 del estatuto del TJUE y 102 de su Reglamento de Procedimiento, carta a la cual, la Comisión no dio respuesta.

${ }^{35} \mathrm{El}$ artículo 260, párrafo 2, TFUE establece que «si la Comisión estimare que el Estado miembro afectado no ha adoptado las medidas necesarias para la ejecución de la sentencia del Tribunal, podrá someter el asunto al Tribunal de Justicia de la Unión Europea, después de haber ofrecido a dicho Estado la posibilidad de presentar sus observaciones. La Comisión indicará el importe de la suma a tanto alzado o de la multa coercitiva que deba ser pagada por el Estado miembro afectado y que considere adaptado a las circunstancias.»

${ }^{36}$ Conclusiones del Abogado general WAHL, N., con respecto a C-95/12, presentadas el 29 de mayo 2013, pt. 32. En el caso contrario, el TJUE hubiese precisado, como lo hizo en los asuntos C-463/04 y C-464/04 (op. cit., pt. 43) que las disposiciones controvertidas constituyen restricciones ilícitas por si mismas o en combinación con la otra disposición en cuestión.

37 Asuntos C-105/12 a C-107/12, op. cit., pt. 45. El TJUE añade que «la interpretación que hace la Comisión de los apartados 48 y 50 de la sentencia Comisión/Alemania, antes citada, se debe a una lectura parcial de dicha sentencia, que no tiene en cuenta los vínculos intrínsecos entre sus diferentes pasajes ni la totalidad y la coherencia de su motivación», pt. 47. 
nas, el gobierno introdujo una enmienda a su ley sobre el Comercio Exterior, el 24 abril $2009^{38}$. A pesar de sus objetivos iniciales, la ley no se aplica solamente a los fondos soberanos, su ámbito es aún más largo ${ }^{39}$. Esta permite al gobierno alemán bloquear las adquisiciones de la empresas alemanas (i) si el adquiriente es una persona que no pertenece a un Estado miembro de la UE, o si éste posee $25 \%$ o más de los derechos de voto, (ii) si después de la transacción, el adquiriente posee directa o indirectamente al menos $25 \%$ de los derechos de voto de la empresa objetivo, y (iii) si la transacción constituye una amenaza para el orden público o la seguridad pública en Alemania ${ }^{40}$.

El órgano competente para actuar es el ministerio de economía. Este puede decidir prohibir las operaciones de adquisición, cuya consecuencia jurídica sería la nulidad del acuerdo de cesión. Si ya han sido transferidas acciones al comprador, el ministerio puede limitar o restringir completamente los derechos de voto en la empresa objeto. No obstante, cabe señalar que la ley prevé, alternativamente, que un inversor puede solicitar al Ministerio un certificado de no-objeción antes de la conclusión de la adquisición. En este caso, es simplemente necesario describir los elementos de base del proyecto de adquisición, el inversor y su ámbito de actividad.

Es preciso señalar que el alcance de la ley puede ser contraproducente. Así pues, la organización del régimen de control no es susceptible de ser calificada de discriminatoria o de restrictiva con respecto a la libre circulación de capitales. La ley requiere un examen riguroso por parte de los inversores extranjeros, que tienen une visibilidad reducida en el margen de maniobra del Estado, mientras que un enfoque más estricto, o al menos más preciso, hubiese sido más adaptado.

También es conveniente no perder de vista que la enmienda de 2009 de la ley, se presenta sobre todo como un mecanismo de repliegue, en el caso en que la última versión de la ley VW sea considerada como contraria al

38 Para un comentario del Außenwirtschaftsgesetz, ver WURTENBERGER, T., y NIEDHARDT, S., «L'Etat actionnaire en Allemagne» en Revue Française d'Administration Publique, n. ${ }^{\circ} 124,2007$, pp. 585 y ss.; PAULLMANN, S., «Patriotisme économique et droit allemand» en VIRASSAMY, G. (coor.), Entreprise et patriotisme économique, L'Harmattan, Paris, 2008.

${ }^{39}$ La ley fue modificada en 2013, ver CASPARY, T., y ECKERT, T., «2013 Reform of Germany's Export Control Act» en Global Trade and Customs Journal, 17 mayo 2013 , pp. 167-172.

${ }^{40}$ Cabe señalar que ninguna obligación de análisis de la transacción es requerida si varios accionistas, provenientes de los Estados no miembros de la UE, disponen en común de $25 \%$ o más de participaciones, en la medida en la que cada uno de ellos, de manera individual, permanezca bajo dicho umbral. Las personas y las empresas de la Asociación Europea de Libre Comercio, es decir, Islandia, Liechtenstein, Noruega, y Suiza, serán tratadas como personas de la UE. 
derecho de la UE ${ }^{41}$. De esta manera, será imposible bloquear la adquisición de títulos de Volkswagen AG, al menos por las empresas de los Estados terceros.

En resumen, existe un movimiento de supresión de las acciones de oro en las economías de los EEMM. En algunos sectores estratégicos, sin embargo, está justificada la conservación de los derechos especiales. Las acciones de oro tienen que ser conformes al derecho de la UE. Estas han dejado progresivamente el lugar a los «poderes de oro», encontrando sus fuentes en las leyes revisadas. Asimismo dicho movimiento gradual de readaptación de los mecanismos nacionales permanece sujeto al control de la Comisión.

\subsection{La continuación del control por parte de la Comisión}

\subsubsection{Continuidad de los controles estrictos de la Comisión}

Como acabamos de mencionar, en el caso alemán con la ley VW, la Comisión está llevando a cabo un esfuerzo de identificación y de control de las posibles infracciones debidas a la persistencia de los derechos especiales con respecto a la libre circulación de capitales y de la libertad de establecimiento.

En este sentido, la posición de determinadas empresas privatizadas griegas es muy interesante. El 29 de enero 2008, Grecia adopto une ley ${ }^{42}$ por la que se establecían dos regímenes de autorización que concernían, por una parte, a la adquisición de acciones con el derecho de voto no rebasando el límite de participación de $20 \%$ y, por otra parte, al poder de decisión en las empresas denominadas estratégicas. Dicha ley prevé (i) que la adquisición de partes de una empresa privatizada, vital para el interés público, superior a $20 \%$ de su capital social, debería ser previamente aprobada por el comité interministerial de privatización, y (ii) algunas decisiones de la empresa, fundamentales para su desarrollo (como su disolución, transformación, fusión o escisión), solamente pueden efectuarse después de la aprobación del ministerio de Finanzas ${ }^{43}$. La Comisión se ha mostrado bastante proactiva. No esperando la eventual aplicación de la ley a una empresa privada, acu-

41 A pesar de la confirmación del TJUE, de la lectura alemana de la sentencia de 2007, la Comisión puede interponer un nuevo recurso por incumplimiento, exclusivamente en contra del mecanismo en vigor.

${ }^{42}$ Ley n. ${ }^{\circ} 3631 / 2008$, Gaceta Oficial, vol. 1 , n. ${ }^{\circ}$ 6, 29 enero 2008.

43 Para un comentario de la legislación, ver PAPADOPOULOS, T., «Greek legislation on strategic investments; the next "golden share" case before the European Court of Justice?» en European Company Law, 2009, pp. 264 y ss. 
dió al TJUE, estimando que había una violación de derecho a la UE, ya que no existía ningún criterio preciso en cuanto a la manera de aplicar dichas disposiciones o en cuanto a las empresas afectadas ${ }^{44}$. En una sentencia del 8 noviembre 2012, el TJUE considera que las disposiciones en cuestión constituyen, efectivamente, un incumplimiento a las obligaciones derivadas del TFUE, en particular de la libertad de establecimiento ${ }^{45}$.

Asimismo, en Grecia, en paralelo a dicho procedimiento, el gobierno decidió aplicar las disposiciones de la ley a la empresa de telecomunicaciones (OTE). En efecto, una empresa, financiada por fondos árabes provenientes de Dubái, compró un porcentaje significativo del capital social de OTE (el 19,9\%). En el Estado griego, que disponía de un porcentaje minoritario en la empresa, cundió el pánico al considerar que dichos fondos, provenientes del exterior del país, estaban a punto de tomar el control de una de las empresas griegas más importantes, crucial para su economía nacional y en cierta medida, para la seguridad pública del país.

Sin embargo, el gobierno griego logró su objetivo. Después de la aprobación de la disposición en cuestión, el fondo árabe decidió cancelar la adquisición de los valores de la sociedad, explicando que no tenía la intención de impugnar la compatibilidad de la disposición griega con el derecho de la UE. Entonces decidió ceder sus participaciones a la empresa de telecomunicaciones alemana Deutsche Telekom. El Estado griego parecía particularmente dispuesto a beneficiarse de dicha empresa como socio estratégico de la OTE. Así pues, la Comisión había pedido al gobierno griego suspender la puesta en marcha de la ley, hasta que su compatibilidad con el derecho de la UE hubiese sido comprobada.

A pesar de esos requerimientos, el gobierno griego procedió a la ejecución de un contrato de cesión de las acciones relativo a 3,03\% de los títulos de la OTE, así como un pacto con los accionistas. Grecia y Deutsche Telekom poseían cada uno 25\% del capital de la OTE, más una acción. El 11 de julio 2008, estos dos acuerdos fueron refrendados por una ley nacional que disponía expresamente, que los acuerdos tenían los mismos efectos que una $\operatorname{ley}^{46}$. Para la Comisión, dichas disposiciones son incompatibles con la libre

44 Seis empresas son afectadas, en particular las que poseen un monopolio en materia de telecomunicaciones, de electricidad, y de agua potable de Atenas y de Tesalónica.

45 Asunto C-244/11, op. cit.

46 Se trata de los siguientes derechos especiales: (i) la designación de las instancias dirigentes de la OTE, incluso como accionista minoritario, (ii) un derecho de veto en toda una serie de dominios concernientes a la estructura y a las actividades de la empresa, y principalmente en materia de disolución y de fusión, de cesión y de conversión de los activos de una empresa estratégica, así como los cambios vinculados a la nacionalidad o al capital, (iii) la aprobación previa de las autoridades griegas para la cesión de acciones de la OTE por Deutsche Telekom. 
circulación de capitales y con la libertad de establecimiento ${ }^{47}$. Después de la sentencia de noviembre 2012, la Comisión espera que esos derechos especiales desaparezcan.

\subsubsection{Regímenes validados por la Comisión}

En ocasiones, la Comisión ha ratificado, expresamente, determinados mecanismos nacionales. Tal es el caso de la ley polaca.

Una ley que contiene un régimen de derechos especiales ${ }^{48}$ en empresas estratégicas ${ }^{49}$ fue adoptada en Polonia en $2005^{50}$. En la práctica, nunca se ha aplicado, en la medida en que el Tesoro del Estado ya contaba con la mayoría del capital social de las entidades en el ámbito de la aplicación de la ley. El control del capital era suficiente para bloquear las decisiones de la empresa que constituían una amenaza para el interés público. No obstante, en 2010, Polonia adoptó une nueva legislación sobre este régimen de derechos especiales que derogaba la anterior ${ }^{51}$.

Dicha modificación fue necesaria después de las discusiones llevadas a cabo con la Comisión. Esta última ponía en tela de juicio la coherencia de las disposiciones de 2005, considerando que eran de orden general, que abarcaban un gran número de sectores y que otorgaban un poder despropor-

47 Ver el comunicado de prensa de la Comisión Europea, IP/12/420 del 26 abril 2012 disponible en Internet, European Commission Press Database Release: http://europa.eu/rapid/ press-release_IP-12-420_en.htm?locale=en (última consulta 18/01/2016).

48 Se trataba del derecho de oposición a determinadas decisiones estratégicas y del de nombrar observadores en las empresas afectadas.

49 Trece empresas afectadas en diversos sectores: la explotación minera de cobre, los medios de comunicación y audiovisuales, la infraestructura ferroviaria, la electricidad y el gas, así como el petróleo.

${ }^{50}$ Ley del 3 junio 2005 relativa a los derechos especiales del Tesoro y sus implicaciones con respecto a las empresas que hubiesen tenido importancia significativa para el orden público o la seguridad pública, Journal of Laws, 2005, n. ${ }^{\circ} 132,1108$.

${ }^{51}$ Ley del 18 marzo 2010 relativa a los derechos especiales del Tesoro y sus implicaciones son respecto a las empresas y grupos de empresas en los sectores de la electricidad, del petróleo y del gas, Journal of Laws, 2010, n. ${ }^{\circ} 65,404$. Conforme al artículo 2 de la Ley, el ministerio del Tesoro puede oponerse a una resolución adoptada por la dirección de la empresa cuyo objeto sea la cesión de activos de una parte de la infraestructura critica, y que amenaza el funcionamiento, la continuidad y la explotación o la integridad de la infraestructura. El ministro del Tesoro tiene también el derecho de oponerse a una resolución del consejo de administración con respecto a la disolución de la empresa, o la supresión de la utilización de una de sus actividades, el cambio del objeto social de la empresa, la venta o la situación geográfica de la empresa. Las objeciones son posibles solo si la aplicación de la decisión constituye una verdadera amenaza para el funcionamiento, la continuidad y la integridad de la infraestructura critica. Las objeciones deben ser emitidas por una autoridad administrativa, cuya decisión pueda ser objeto de un recurso administrativo. 
cionado al Tesoro en cuanto a los acuerdos importantes de las sociedades ${ }^{52}$. En este contexto, resulta interesante resaltar la posición tomada por la Comisión con respecto a la nueva ley. Pareciera que para ésta, en efecto, dicho acto constituía una «solución modelo» ${ }^{53}$ a la cuestión de compatibilidad de los regímenes de los derechos especiales de los EEMM con el derecho de la UE. La ejemplaridad de dicha ley significa que ésta podría ser copiada, o al menos, servir de inspiración, a los otros EEMM.

Un análisis de las principales disposiciones de la ley nos lleva sin embargo a conclusiones sorprendentes. Los comentaristas consideran así que el régimen de los derechos especiales establecido, tiene muchos defectos y es imperfecto, ya que la aplicación precisa y justa del mecanismo necesita una ley suplementaria ${ }^{54}$. Mientras que en el caso de la ley de 2005 , se criticaba la falta de necesidad real, en el caso de la ley 2010, se criticaba el contenido del acto normativo.

En efecto, su ámbito de aplicación estaba particularmente mal definido, lo que podría llevar a las empresas afectadas a preguntarse si tal ámbito de aplicación era real. Las disposiciones de la ley también podrían interpretarse de manera que pudieran ser eludidas por las empresas. Además, se planteaban dudas en el marco de dicha ley sobre la posibilidad real para el Estado polaco de asegurar la seguridad pública, en lo que respecta a las empresas que actúan en sectores estratégicos y en particular de la definición excesivamente restrictiva del alcance del derecho de veto. Asimismo, el plazo de reacción previsto para resolver las cuestiones sujetas al derecho de veto no permitiría efectuar un análisis completo de los posibles problemas aplicables.

Existen demasiadas incertidumbres para considerarla un modelo de legislación para los otros EEMM. Fundamentalmente, porque la ley, en su forma actual, carece de fuerza vinculante ${ }^{55}$, poniendo en entredicho la oportunidad de tal acto normativo.

En definitiva, el intenso trabajo de la Comisión no parece disminuir, sensación que corroboran los mecanismos alemán y griego. Los EEMM tratan de eludir, anticipar o evitar los controles efectuados por la Comisión. Así pues, los esfuerzos, realizados tanto por los EEMM como por la Comi-

52 Ver el comunicado de prensa de la Comisión Europea, IP/09/1634 del 29 de octubre de 2009 disponible en Internet, European Commission Press Database Release: http://europa.eu/ rapid/press-release_IP-09-1634_en.htm?locale=en (última consulta 18/01/2016).

53 GRZEGORCZYK, F., «The New Polish Law on the "Golden Veto": A Model Act for Member States of the European Union?» en European Company Law, n. ${ }^{\circ}$ 9, 2012, p. 21.

${ }_{54}$ Ibid., p. 23, y ADAMCZYK, L., y BARAŃSKI, T., «The Compatibility of the Polish State's Golden Shares with EC Law» en European Business Organization Law Review, n. ${ }^{\circ} 11,2010$, pp. 95 y ss.

55 Ibid., p. 25. 
sión, permiten en ocasiones reconocer como adecuados a ciertos regímenes de los derechos especiales. Para armonizar dichas posiciones en materia de las acciones de oro, sería necesario que la UE adoptara medidas dirigidas a todos y cada uno de los EEMM, en su función de accionista especial de las empresas.

\section{La necesidad de una directiva sobre las acciones de oro}

Para los EEMM es delicado aplicar los derechos especiales en las empresas explicando al mismo tiempo que están obligados a doblegarse a los imperativos del mercado único funcional de la UE. La conciliación de exigencias tan heterogéneas como la salvaguardia de intereses esenciales en determinadas empresas o sectores y la continua expansión de dicho mercado interior, requieren una intervención a nivel de la UE.

Ahora bien, si hemos mencionado la condena recurrente de las acciones específicas por parte de las instituciones de la UE, es pertinente señalar que éstas últimas otorgan, sin embargo, a los EEMM, siempre y cuando se cumplan determinadas modalidades en ámbitos bien definidos, la posibilidad de utilizar los derechos especiales (1). Esta situación invita a reflexionar más profundamente sobre la oportunidad de una normativa de derecho derivado reglamentando la práctica de las acciones de oro. Si tal propuesta se ha señalado algunas veces ${ }^{56}$, trataremos de sentar realmente las premisas que podrían constituir el contenido (2).

\subsection{La oportunidad de una directiva de las acciones de oro}

\subsubsection{Acciones de oro organizadas por el derecho de la UE}

El derecho de la UE condena los derechos especiales si constituyen restricciones que no estén justificadas como excepciones al TFUE o por razones imperiosas de interés general, y que no son proporcionadas en relación con los objetivos perseguidos. Ya lo hemos explicado previamente: no

56 Ante las preocupaciones de los Estados miembros, Peter Mandelson, anterior miembro de Comisión encargado del Comercio exterior, explicó en 2008 que «la emergencia de los fondos soberanos es un fenómeno nuevo cuestionable, sobre todo si estas inversiones se realizan con intereses políticos. Nuestra respuesta inmediata no deberá prohibirlos. Por otro lado, será difícil definir sectores estratégicos dentro de la Unión Europea. Debemos imaginar mecanismos que garanticen el control europeo sobre sectores vitales, como una acción de oro europea.» 
existe une sentencia per se de un mecanismo parecido. No solamente dichos mecanismos son aceptables, sino que incluso el derecho derivado de la UE prevé en ciertas hipótesis muy limitadas tales prácticas.

\subsubsection{Abastecimiento energético}

En términos de abastecimiento energético, por ejemplo, las instituciones de la UE consideran que las redes de transporte de gas y electricidad, son esenciales para la seguridad pública y la competitividad de la UE. Las medidas de salvaguardia son, pues, esenciales para preservar la seguridad del abastecimiento energético de la UE. El problema ha surgido por la voluntad del gigante ruso Gazprom de tomar control de las principales empresas de la UE. En dicho contexto, la Comisión ha estimado que no se les debería permitir a las empresas de Estados terceros obtener el control de una red de transporte o un gestor de la red, en caso de falta de conformidad por no cumplir con los requisitos exigidos por la UE. Sin embargo, el problema del abastecimiento energético debe tener en cuenta los acuerdos internacionales entre la UE y el tercer país de que se trate.

Las directivas de gas y electricidad del 13 de julio de 2009 establecen que las autoridades reguladoras nacionales, bajo el control de la Comisión, podrán denegar el acceso al mercado a los propietarios o gestores del sistema de transporte en el que una o más personas de los Estados terceros ejerzan el control en caso de amenaza a la seguridad del abastecimiento de energía de los EEMM de la UE ${ }^{57}$. El TJUE ha tenido ocasión de pronunciarse sobre el contenido de dichas directivas en una reciente cuestión prejudicial. En la sentencia del 22 de octubre de 2013, se precisó que los objetivos para garantizar la seguridad del abastecimiento de energía por un lado, y el mantenimiento de una competencia no falseada para proteger a los consumidores, por otro, son razones imperiosas de interés general. En este sentido, las medidas en cuestión, la prohibición de la privatización y la prohibición del grupo, restringen la libre circulación de capitales, pero son conformes a la legislación de la $\mathrm{UE}^{58}$.

57 Directiva 2009/72/CE del Parlamento Europeo y del Consejo, de 13 de julio de 2009 , sobre normas comunes para el mercado interior de la electricidad y por la que se deroga la Directiva 2003/54/CE DO L 211, p. 55; y directiva 2009/73/CE del Parlamento Europeo y del Consejo, de 13 de julio de 2009, sobre normas comunes para el mercado interior del gas natural y por la que se deroga la Directiva 2003/55/CE, DO L 211, p. 94. La versión inicial de los textos de la Comisión preveía una prohibición absoluta. Finalmente, el procedimiento previsto es de una prohibición caso por caso, por cada Estado miembro.

58 Asuntos C-105/12 a C-107/12, op. cit., pts. 48, 66 y 67. 


\subsubsection{Transporte aéreo de pasajeros}

En materia de transporte aéreo de pasajeros, las instituciones de la UE, como algunos Estados terceros, también niegan el control de las líneas aéreas de la UE por parte de empresas de Estados terceros. En este sentido, el artículo 4 del Reglamento (CEE) n. ${ }^{\circ} 2407 / 92$ del Consejo, de 23 de julio de 1992, sobre las licencias a las compañías aéreas, es particularmente claro. Precisa que para recibir una licencia, una compañía aérea « deberá ser propiedad y continuar siéndolo, directamente o mediante la propiedad mayoritaria, por los EEMM y/o los ciudadanos de los EEMM. Esta deberá, en todo momento, poder ser controlada por dichos Estados o por dichos ciudadanos» ${ }^{59}$.

\subsubsection{Defensa}

En otro orden de cosas, las directivas del « paquete de defensa $»^{60}$, que representan un primer paso hacia la apertura del sector del armamento a las reglas del libre mercado, se tuvo que tener en cuenta las particularidades de cada Estado miembro, y en este sentido, tratar de no obstaculizar el orden público o la seguridad pública. Toda transferencia de productos relacionados con la defensa es objeto de una autorización previa, a través de una licencia general de transferencia, global o individual, expedida por el Estado miembro a partir del cual el proveedor pretende transferir productos relacionados con la defensa. Por otra parte, los EEMM disponen de la posibilidad de discriminar a los destinatarios de las licencias, si fuese necesario, por razones de protección de los intereses esenciales de su seguridad.

59 Reglamento (CEE) 2407/92 del Consejo, de 23 de julio de 1992, sobre las licencias de las compañías aéreas, DO L 240 de 24 de Augusto de 1992, p. 1; y ver, también sobre los acuerdos Open Skies, TORRENT, R., «Pourquoi un revirement de la jurisprudence "golden share" de la Cour de l'Union européenne est-il indispensable ?» en SANTACRUZ, M. (coor.), A Man for All Treaties : liber amicorum en l'honneur de Jean-Claude Piris, Bruylant, Bruxelles, 2012, pp. 539 y ss.

${ }^{60}$ Directiva 2009/81/CE del Parlamento Europeo y del Consejo, del 13 julio 2009, sobre coordinación de los procedimientos de adjudicación de determinados contratos de obras, de suministro y de servicios por las entidades o poderes adjudicadores en los ámbitos de la defensa y la seguridad, y por la que se modifican las Directivas 2004/17/CE y 2004/18/CE, DO L 216, p. 76; Directiva 2009/43/CE del Parlamento Europeo y del Consejo, de 6 de mayo de 2009 , sobre la simplificación de los términos y las condiciones de las transferencias de productos relacionados con la defensa dentro de la Comunidad, DO L 146, p. 1. Dichos textos tienen como objetivo limitar las veleidades proteccionistas de los gobiernos en la adjudicación de los contratos públicos de defensa. 


\subsubsection{Telecomunicaciones}

Por último, puede considerarse que la Comisión se propone iniciar un mercado único de telecomunicaciones, armonizando principalmente la política del espectro radioelectrico ${ }^{61}$. La idea consiste en conceder a las empresas de telecomunicaciones de un «pasaporte» válido en toda Europa. Ahora bien, determinados EEMM entre los que figuran Alemania, Francia y Gran Bretaña, se resisten a ceder el control de las subastas del espectro. Dichos países consideran que ésta materia forma parte del ámbito de seguridad nacional. Las discusiones entre los gobiernos y la Comisión están siendo muy animadas, y posiblemente ésta última tenga que modificar su proyecto para incluir un determinado número de condiciones y limitaciones en el ámbito de las prerrogativas especiales de los EEMM mencionados.

Aunque los poderes acordados a los EEMM por parte de la Comisión parecen estar muy restringidos, su existencia tranquiliza a veces a los gobiernos en cuanto al control de sus políticas. Ahora bien, algunos desearían ir más lejos y enmarcar estos diferentes poderes en una directiva., Se trataría de establecer un marco jurídico preciso que permitiera armonizar la posición de los EEMM, dejándoles cierto margen de flexibilidad en la aplicación de los derechos especiales.

\subsection{La aplicación de una directiva sobre las acciones de oro}

\subsubsection{Competencia}

Poner en marcha una normativa al nivel de la UE que establezca un marco de aplicación concerniente a la utilización de los derechos especiales por los EEMM, exigiría, en primer lugar, interrogarse sobre el alcance de la competencia de las instituciones de la Unión en la materia. En efecto, hemos observado que los EEMM consideran que dichos derechos enmarcan intereses esenciales, y en este sentido, que se consideran los más indicados para actuar en la materia. Asimismo, es necesario recordar que tales poderes limitan el funcionamiento del mercado interior. Sin embargo, éste

61 Ver los intercambios en el sitio de Internet de la Comisión, a raíz de la propuesta del espectro radioeléctrico, COM/2010/471 final, disponible en Internet, Comisión Europea: http://www.europarl.europa.eu/meetdocs/2009_2014/documents/com/ com_com(2010)0471_/com_com(2010)0471_es.pdf (última consulta 18/01/2016). Cabe recordar que el espectro eléctrico corresponde a las ondas radioeléctricas (ondas electromagnéticas que se propagan en el espacio sin guía artificial) cuya frecuencia es de entre 9 khz y 3000 Ghz. 
es uno de los ámbitos en los cuales la UE dispone de una competencia compartida con los EEMM ${ }^{62}$.

En este sentido, una acción a nivel de la UE significaría que los EEMM ya no podrían actuar a discreción, o solamente si la UE no hubiese ejercido su competencia. La elección de una política tal provoca reticencias en los EEMM, para quienes la vulnerabilidad de los derechos especiales se percibirá como una amenaza a la soberanía. Asimismo, la Comisión puede dudar en comprometerse en una iniciativa que estará claramente en contra de la política que ellamisma está llevando a cabo, pues, de conformidad con el artículo 50 TFUE, le corresponde a ella promover y presentar una propuesta de acto legislativo al Parlamento europeo y al Consejo ${ }^{63}$.

\subsubsection{Armonización positiva}

Una iniciativa de esa envergadura se ha vuelto no obstante indispensable, dado que en materia de acciones específicas las sentencias a veces equívocas del TJUE topan con sus límites. Por eso es importante pasar del marco de armonización negativa del derecho de la UE a su armonización positiva $^{64}$. Una iniciativa de la UE deberá seguir un objetivo doble: por una parte, armonizar los mecanismos de control de los EEMM en las empresas privatizadas, garantizándoles la protección y eficacia de sus intereses nacionales estratégicos; y por otra parte, mejorar el desarrollo de la competitividad de las empresas ofreciéndoles un entorno jurídico más transparente y previsible para los inversores potenciales.

Con la finalidad de que la acción de la UE pueda alcanzar los objetivos fijados, ésta deberá ser lo más sencilla posible. El instrumento jurídico adecuado para una legislación sobre los derechos especiales podría entonces ser una directiva, más que un mecanismo bastante detallado, lo que permitiría respetar las exigencias del TFUE, siempre respetando un margen de maniobra decisoria en el plan nacional. Ahora bien, por las razones mencionadas anteriormente, el procedimiento legislativo ordinario corre el riesgo de fracasar. La alternativa seria entonces inspirarse en la misma iniciativa que se utilizó para concebir las reglamentaciones del sector de finanzas, el llamado proceso Lamfalussy.

62 Artículo 4 TFUE.

${ }^{63}$ Se trata del procedimiento legislativo ordinario.

${ }^{64}$ Sobre las nociones de armonización negativa y positiva, ver, por ejemplo, DOUGAN, M., «Minimum Harmonisation and the Internal Market» en Common Market Law Review, n. ${ }^{\circ} 4,2000$, pp. 853 y ss. 


\subsubsection{El proceso Lamfalussy}

El proceso Lamfalussy, iniciado en 2001, pretende, por una parte, poner en marcha un mecanismo eficaz de convergencia en las prácticas de vigilancia financiera a escala de la UE y, por otra parte, permitir a la legislación de la UE sobre los servicios financieros actuar rápidamente y de manera flexible para cambiar con la evolución de los mercados financie$\operatorname{ros}^{65}$. Dicho proceso reglamentario está fundado en cuatro niveles. El primer nivel es el de la toma de decisión. La Comisión hace una propuesta de directiva al Parlamento y al Consejo europeo que se pronuncian únicamente sobre los grandes principios. En el segundo nivel, un comité especializado desarrolla, en colaboración con las autoridades de regulación de los EEMM, los aspectos técnicos de la aplicación de la legislación en cuestión. Dichos intercambios se efectúan con perfecta transparencia respecto del Parlamento. En el tercer nivel, las autoridades de regulación nacionales coordinan, en una red, la aplicación más adecuada de la legislación de la UE, con la finalidad de que las reglamentaciones de los EEMM difieran lo menos posible. En el cuarto nivel, la Comisión desempeña plenamente su papel de guardiana de los tratados, verificando el respeto de dichos textos por los EEMM, y velando por su aplicación por medio del recurso al TJUE, si fuese el caso ${ }^{66}$. Aunque no haya quedado exento de críticas ${ }^{67}$ dicho proceso ofrece ventajas en materia de flexibilidad y reactividad reglamentaria, puede ser aplicado a materias distintas de los servicios financieros $y$, en particular, a las acciones de oro.

\subsubsection{Aplicación en el marco de una directiva de las acciones de oro}

La idea aquí no es instaurar una acción específica de la UE, sino de proponer une procedimiento apropiado que autorice la aplicación de las acciones de oro en cada Estado miembro.

65 Sobre este proceso, ver, por ejemplo, VACCARI, B., «Le processus Lamfalussy: enjeux, leçons et perspectives» en Revue du Droit de l'Union Européenne, n. ${ }^{\circ}$ 1, 2007, pp. 41 y ss.

66 Ver Premier Rapport du Comité des Sages sur la régulation des marchés européens de valeurs mobilières, 9 noviembre 2000, disponible en Internet, Comisión Europea: http:// ec.europa.eu/finance/securities/docs/lamfalussy/wisemen/final-report-wise-men_fr.pdf (última consulta 18/01/2016).

67 Ver, por ejemplo, SOUSI-ROUBI, B., «La procédure Lamfalussy à l'épreuve de la directive concernant les marchés d'instruments financiers» en Euredia, n. ${ }^{\circ}$ 2, 2004, pp. 209 y ss.; y POSNER, E., «The Lamfalussy process: polyarchic origins of networked financial rulemaking in the EU» en SABEL, C., y ZEITLIN, J., Experimentalist governance in the European Union, Oxford University Press, Oxford, 2010, pp. 43 y ss. 
En primer lugar, en el primer nivel, una directiva marco deberá definir las orientaciones generales regulando la creación de acciones específicas en los EEMM de la UE. Esto permitiría, en particular, impedir el uso del mecanismo por razones relativas al mercado interior, evitando así que los EEMM impongan medidas proteccionistas intracomunitarias. Si este primer nivel parece a primera vista simple en cuanto a la aplicación, falta un consenso acerca del marco general de armonización. Este primer nivel es indispensable ya que deberá reactivar une convergencia de políticas nacionales con la jurisprudencia y la práctica de la UE. La directiva deberá también definir un ámbito de aplicación claro, abarcando los sectores en los cuales evolucionan empresas estratégicas.

Así, por ejemplo, el sector de la defensa deberá estar protegido por razones de seguridad, pero los EEMM han de poder mantener derechos, aunque limitados, en las empresas de energía, transporte, telecomunicaciones y salud. La directiva podrá inspirarse de la jurisprudencia en la materia y también de la directiva Infraestructuras Críticas Europeas ${ }^{68}$.

Esta última define los sistemas localizados en los EEMM considerados como indispensables para el mantenimiento de las funciones vitales de la sociedad, de la salud, de la garantía, de la seguridad y del bien estar económico o social de los ciudadanos, y cuyo paro o destrucción tendrá un impacto significativo en un Estado miembro. Dicha directiva pretende, principalmente, hacer frente al terrorismo. Ahora bien, en materia de acciones específicas, los Estados no dudan en justificar sus poderes sobre la base de una lucha combinada contra el terrorismo y los fondos soberanos ${ }^{69}$. La propugnada directiva sobre las acciones de oro podría inspirarse de esta otra directiva con la finalidad de proteger ese tipo de intereses vitales y esenciales de cada Estado miembro.

La directiva debería establecer además una tipología de los derechos especiales en cuestión. La práctica muestra que pueden utilizarse los derechos de veto absolutos, a posteriori, sobre las estructuras accionarias o las decisiones de la sociedad, la nominación de miembros del consejo de administración, o incluso el aumento del poder de voto de un título. La directiva deberá también prever obligaciones de transparencia y de previsibilidad, es decir de seguridad jurídica, en contra de los inversores actuales o potenciales. Así pues, la directiva debería inspirarse de los textos en materia de energía, mencionados anteriormente, en relación con dos aspectos: en primer lugar, podría establecer una inversión de la carga

${ }^{68}$ Directiva 2008/114/CE del Consejo, de 8 de diciembre de 2008, sobre la identificación y designación de infraestructuras críticas europeas y la evaluación de la necesidad de mejorar su protección, DO L 345, p. 75.

69 Ver supra las justificaciones del Gobierno griego en Asunto C-528/10. 
de la prueba. En efecto, mientras que generalmente son los EEMM quienes, limitando las libertades de circulación, han de probar la necesidad y la proporcionalidad de las restricciones, se trataría aquí de prever que corresponde al inversor potencial demostrar la ausencia de riesgo para la seguridad y el orden público. Aunque pueda parecer restringienda, la inversión de la carga de la prueba permitiría aclarar la estrategia del inversor, en lugar de basarse en suposiciones de los EEMM. En segundo lugar, la directiva debería permitir «el levantamiento del velo corporativo». Con el fin de identificar la existencia de la gravedad de una amenaza, la idea aquí consiste en buscar la nacionalidad de la entidad que controla, en última instancia, al operador que se propone invertir en las empresas de los EEMM. Muchos inversores extranjeros abusan del law shopping, creando un efecto de entidades jurídicas relativamente vacías en el territorio de un Estado miembro con el objetivo de beneficiarse de las normas del TFUE sin conformarse plenamente a determinadas normas de la UE. El interés es determinar si deberían aplicarse dichas normas en materia de inversiones intracomunitarias o con Estados terceros.

Posteriormente, en un segundo nivel, la negociación de los términos y de las condiciones, así como la aplicación de las acciones de oro (y principalmente de los derechos especiales que están vinculados con éstas) se delegaría en los EEMM más afectados por las operaciones en cooperación con un comité de reguladores. Este estaría instituido por una decisión de la Comisión, y compuesto por representantes de los EEMM. Dicho comité debería ser consultado por la Comisión durante la elaboración de las propuestas legislativas sobre las cuestiones relativas a las acciones de oro. Cada Estado miembro designaría una autoridad administrativa competente que desempeñaría las obligaciones de la directiva y que controlaría la aplicación de los mecanismos atinentes a los derechos especiales. Estas autoridades competentes serían plenamente independientes de los operadores del mercado. La Comisión podría validar las condiciones para la expedición de una acción de oro tras el examen de su conformidad con la legislación de la UE.

En el tercer nivel, el comité de reguladores tendría como misión armonizar las normas de aplicación y aconsejar a la Comisión sobre las medidas necesarias para mantener y adaptar la legislación en función de la evolución política y económica del mercado. Las autoridades competentes de varios EEMM deberían cooperar entre ellas, en particular, intercambiar información sobre las actividades de investigación o de supervisión. Para facilitar dicha cooperación, los EEMM designarían una autoridad competente única que serviría de punto de contacto para los fines de la directiva, que podría ser el comité de reguladores (o una subdivisión de dicho organismo). 
Finalmente, en el cuarto nivel, los EEMM deberían notificar y comunicar a la Comisión, y a los otros EEMM, los derechos especiales queestablezcan. La Comisión podrá tomar medidas con la finalidad de que se respete la legislación en caso de infracción contra el derecho de la UE.

En definitiva, una directiva marco relativa a las acciones específicas permitiría proteger a las empresas estratégicas mediante una política armonizada al nivel de la UE, sin sustituir a los EEMM. No obstante, esto exige una construcción profunda previa de la UE por la «unionización» de los intereses actualmente nacionales. Dicho enfoque respaldaría el papel del Estado miembro, como accionista especial, en la protección de las empresas estratégicas contra las tomas de posesión extranjera.

\section{Conclusión}

Los EEMM han tratado de reinventar las modalidades de los derechos especiales vinculados con las acciones de oro, con la finalidad de proteger sus empresas nacionales y de permitirles desarrollarse a nivel internacional. Si ya no actúan principalmente por la puesta en marcha de las acciones específicas, habiendo encontrado alternativas al mecanismo, una intervención de la UE permitiría armonizar las diferentes políticas «patrióticas» que han emergido en los EEMM.

Para conceder una verdadera dimensión transfronteriza a las empresas que evolucionan en los sectores esenciales, el esfuerzo debería venir del nivel supranacional. Dicha voluntad permitiría reforzar el peso económico de la UE frente a nuevas potencias extranjeras. Sin embargo, es preciso constatar el fracaso actual de las instancias de la UE en la gestión de los grandes grupos multinacionales. Esto se explica por la ausencia de visión estratégica propia, resultante, en parte, de una falta de autoridad política de la UE suficientemente vigorosa en la materia, capaz de rebasar los intereses nacionales de cada Estado miembro, y actuar en el interés común.

El fracaso es doble. No solamente los EEMM no logran ponerse de acuerdo sobre el contenido de los sectores estratégicos afectados ni sobre los intereses que hay que preservar sino que, además, el principio de la protección de intereses estratégicos comunes, en el marco de determinadas inversiones extranjeras, encuentra un obstáculo adicional en la identificación del órgano de la UE, susceptible de encarnar dichos intereses. En ausencia de voluntad política sobre la implementación de un mecanismo común, los EEMM de la UE que aspiran a defender sus intereses nacionales, permanecen sujetos al control de las instituciones europeas. 
En una Europa de «pequeños pasos», dicho inmovilismo puede ser muy perjudicial con respecto a los Estados y las Empresas extrajeras de la UE. Los EEMM de la UE deberán avanzar juntos para aprovechar la cohesión y la puesta en común de sus políticas. Ahora bien, el único y verdadero nivel de respuesta adecuado para superar la crisis financiera actual es el supranacional. 


\section{Derechos de autor (Copyright)}

Los derechos de autor de esta publicación pertenecen a la editorial Universidad de Deusto. El acceso al contenido digital de cualquier número de Cuadernos Europeos de Deusto (CED) es gratuito, transcurridos 6 meses desde su publicación. Los trabajos podrán descargarse, copiar y difundir, sin fines comerciales y según lo previsto por la ley. Así mismo, los trabajos editados en CED pueden ser publicados con posterioridad en otros medios o revistas, siempre que el autor indique con claridad y en la primera nota a pie de página que el trabajo se publicó por primera vez en CED, con indicación del número, año, páginas y DOI (si procede). 